\title{
Intención y práctica de actividad física en maestros españoles
}

\author{
Intentions and practice of physical activity in spanish teachers
}

\section{Intenção e atividade física em mestres espanhóis}

\author{
Blázquez Manzano, A ${ }^{1}$. Ana León-Mejía, A. ${ }^{2}$ y Feu Molina, S. ${ }^{3}$ \\ ${ }^{1}$ Universidad Camilo José Cela, (España), ${ }^{2}$ Universidad Internacional de La Rioja (España) y Open University (UK) y ${ }^{3}$ Universidad de Extremadura (España)
}

Resumen: El objetivo de este trabajo fue comparar la práctica actividad física semanal y la intención de mantenerse físicamente activos en maestros de educación infantil y primaria, así como conocer si les condiciona en estos aspectos su situación personal y laboral. Los participantes de este estudio fueron 415 maestros, 162 hombres y 353 mujeres. Se adaptó y validó, a través de una análisis factorial y confirmatorio, el cuestionario Intention to be Physically Active de Hein, Müür y Koka (2004) para una población de adultos en el ámbito laboral, alcanzado una fiabilidad alta $(\alpha=.91)$. El análisis inferencial mostró que la mujeres hacen menos actividad física que los hombres y que en la práctica habitual de actividad física y en la intención de ser físico activo los/as docentes solteros/as y sin hijos puntúan más alto que los casados y con hijos. Los maestros y maestras que más actividad física realizan presentan una mayor intención a realizar actividad física en el futuro, y estos resultados se interpretan en relación al tiempo de ocio que tienen los participantes en este estudio y las variables sociales que inciden en el mismo.

Palabras clave: Maestros de Primaria e Infantil, Nivel práctica física, Intención de práctica física

Abstract: The main goal of this paper is to compare intention of being physically active with actual weekly practice of physical exorcize among 451 preschool and primary teachers who participated in this study (162 males and 353 females). We explore this topic in relation to social and labour variables. The adapted and validated Spanish version of the Intention to be Physically Active Scale (Hein, Müür and Koka, 2004) indicated high internal consistency $(\alpha=.91)$. Further inferential analyses showed that women engage less in physical activity compared to men. Also, single teachers without kids scored higher in both intention and actual practice of physical activity in comparison with those who are married and have kids. Intention and practice are correlated and these results are interpreted in terms of differences in leisure-time, as well as in terms of social variables related to spare time.

Key words: Preschool and Primary teachers, Physical activity and Intention of being Physically Active

Resumo: O objetivo deste trabalho foi comparar a prática atividade física semanal e a intenção de se manter fisicamente activos em professores de ensino infantil e básico, alé de conhecer se lhes condiciona nestes aspetos a sua situação pessoal e laboral. Os participantes deste estudo foram 415 professores, 162 homens e 353 mulheres.

Adaptou-se e validou, através de uma análise factorial e confirmatorio, o inquérito Intention to bê Physically Active de Hein, Müür e Koka (2004) para uma populaçáo de adultos no âmbito laboral, atingido uma fiabilidade alta $(\alpha=.91)$.

A análise inferencial mostrou que mulheres fazem menos atividade física do que os homens e que na prática habitual atividade física e na intenção de ser físico ativo os/as professores solteiros/as e sem filhos pontuan mais alto do que os casados e com filhos.

Os profesores/as que mais atividade física realizam apresentam uma maior intenção a realizar atividade física no futuro, e estes resultados interpretamse em relação ao tempo de lazer que têm os participantes neste estudo e as variáveis sociais que incidem no mesmo.

Palabras claves: Profesores de Ensino e Básico, Nível prática física, Intenção de prática física.

\section{Introducción}

Distintas investigaciones epidemiológicas, correlacionales y experimentales, así como un amplio número de meta-análisis, han puesto de manifiesto que la realización de actividades físicas con regularidad influye positivamente en el estado de salud, tanto físico como mental (Caracuel y Arbinaga, 2010; Fox, 1999; Ramírez, Timón, Olcina, Muñoz, Robles y Maynar, 2012; Sofi, Capalbo, Cesari, Abbate y Gensin, 2008; Nocon, Hiemann, Müller-Riemenschneider, Thalau, Roll y Willich, 2008). Del mismo modo, también se ha subrayado que llevar una vida sedentaria es poco saludable, ya que aumenta el riesgo de padecer afecciones y dolencias que la Organización Mundial de la Salud (OMS) cataloga dentro del grupo de enfermedades no transmisibles o ENT, tales como

Dirección para correspondencia [Correspondence address]: Alberto Blázquez Manzano. E-mail: albertoblazqu50@gmail.com la diabetes, hipertensión, enfermedades coronarias, respiratorias, óseo-musculares, metabólicas, obesidad, accidentes cerebrovasculares, depresión e incluso cáncer. En concreto, la inactividad física es el cuarto factor de riesgo más importante de mortalidad en todo el mundo. Además, es también la causa principal de un 21-25\% de los cánceres de mama y de colon, del $27 \%$ de la diabetes, y del $30 \%$ de las cardiopatías isquémicas (OMS, 2009; 2010). Por el contrario, mantener unos niveles razonables de actividad disminuye el riesgo de padecer enfermedades crónicas o de una muerte prematura.

Todos estos datos son preocupantes a la luz de la ultima encuesta de Salud en Espańa realizada entre el 2011 y el 2012, según la cual el $41 \%$ de los individuos mayores de 18 ańos se declara sedentario (INE, 2013). La ciudadanía espańola tiene un estilo de vida eminentemente sedentario y esto choca con 
el hecho de que las campañas preventivas, informativas y publicitarias sobre la relación entre salud y actividad física son continuas (Moscoso, Serrano, Biedma y Marfin, 2013). Estas campañas han permitido sensibilizar a gran parte de la sociedad española, con lo cual cabe preguntarse, ¿a qué se debe esta inactividad física? Se desconoce si se trata de un mero problema relacionado con la falta de motivación o intención de mantenerse activo o si, por el contrario, se relaciona con la falta de tiempo que interfiere con una inclinación favorable a realizar ejercicio físico.

En este trabajo se explora la intención de mantenerse físicamente activo en una muestra de maestros de Espańa de varias especialidades, examinando distintos factores personales y laborales. Para ello, se compara el grado de actividad física semanal de los maestros de infantil y de educación primaria con la intención que tienen de mantenerse físicamente activos. El objetivo último es examinar en qué medida existe una discrepancia entre realidad e intención, y qué posibles causas pueden atribuirse a la misma.

\section{Método}

El diseño de este estudio es una investigación instrumental, en la medida que se adapta y analizan las propiedades psicométricas de un instrumento en una población muy diferente de la original; y un estudio comparativo por cuanto indaga sobre las diferencias que se producen en la práctica física y en la intención de ser activo por la situación sociodemográfica y laboral de los participantes (Ato, López y Benavente, 2013).

\section{Participantes}

Los participantes de este estudio fueron 415 maestros de educación primaria e infantil, 162 hombres y 353 mujeres. Las características de los participantes recogidas a través de las variables género, rango de edad, estado civil, número de hijos, tipo de centro, tipo de contrato laboral, años de experiencia profesional y especialidad que imparte como docente se presentan en la tabla 1.

Tabla 1. Características de la muestra.

\begin{tabular}{llcc}
\hline Variables & & $N$ & $\%$ \\
\hline Género & a. Hombre & 162 & 31,46 \\
\multirow{3}{*}{ Edad } & b. Mujer & 353 & 68,54 \\
\cline { 2 - 4 } & a. $<25$ años & 29 & 5,71 \\
& b. $26-40$ años & 287 & 56,50 \\
& c. $41-55$ años & 155 & 30,51 \\
\multirow{2}{*}{ Estado Civil } & d. $<55$ años & 37 & 7,28 \\
\cline { 2 - 4 } & a. Soltero & 184 & 35,73 \\
& b. Casado/Pareja & 307 & 59,61
\end{tabular}

\begin{tabular}{|c|c|c|c|}
\hline Variables & & $N$ & $\%$ \\
\hline & c. Divorciado & 19 & 3,69 \\
\hline & d. Viudo & 5 & 0,97 \\
\hline \multirow[t]{2}{*}{ No hijos } & a. Sin hijos & 266 & 51,65 \\
\hline & b. Con hijos & 249 & 48,35 \\
\hline \multirow[t]{2}{*}{ Tipo de centro } & a. Público & 367 & 71,26 \\
\hline & b. Concertado & 148 & 28,74 \\
\hline \multirow[t]{5}{*}{ Estatus laboral } & a. Funcionario & 249 & 48,35 \\
\hline & b. Interino & 103 & 20,00 \\
\hline & c. Cooperativista & 7 & 1,36 \\
\hline & d. Contrato indefinido & 116 & 22,52 \\
\hline & e. Contrato temporal & 40 & 7,77 \\
\hline \multirow[t]{4}{*}{ Experiencia } & a. $<$ 1año & 17 & 3,30 \\
\hline & b. De 1 a 5 años & 136 & 26,41 \\
\hline & c. De 5 - 10 años & 122 & 23,69 \\
\hline & d. $>10$ años & 240 & 46,60 \\
\hline \multirow[t]{8}{*}{ Especialidad } & a. Ed. Infantil & 99 & 19,22 \\
\hline & b. Ed. Primaria & 182 & 35,34 \\
\hline & c. Ed. Musical & 29 & 5,63 \\
\hline & d. Ed. Física & 58 & 11,26 \\
\hline & e. Ed. Especial & 33 & 6,41 \\
\hline & f. Lenguas extranjeras & 72 & 13,98 \\
\hline & g. Audición y Lenguaje & 18 & 3,50 \\
\hline & h. Religión & 24 & 4,66 \\
\hline
\end{tabular}

\section{Instrumento}

En este trabajo se ha utilizado la Medida de intencionalidad para ser Físicamente Activo (MIFA). Esta escala fue inicialmente adaptada al castellano por Moreno, Moreno y Cervelló (2007), a partir de la escala Intention to be Physically Active de Hein et al. (2004). La escala está compuesta por cinco ítems que miden la intención del sujeto de ser físicamente activo en su vida adulta, tras su formación educativa. Los ítems van precedidos de la frase "Respecto a tu intención de practicar alguna actividad fisico-deportiva...". Las respuestas corresponden a una escala tipo Likert que oscila de 1 a 5 , donde 1 corresponda a "totalmente en desacuerdo" y 5 a "totalmente de acuerdo".

Este cuestionario se ha utilizado con estudiantes de educación primaria (Arias, Yuste y Castejón, 2013), educación secundaria (Moreno et al. 2007), jugadores de baloncesto entre 11 y 16 años (Franco, Pérez-Tejero y Arrizabalaga, 2012), estudiantes universitarios (Expósito, Fernández, Almagro, Sáenz-López, 2012). Esta escala no se ha empleado en adultos y, por ende, en maestros. Sin embargo, al ser estos últimos un ejemplo para los escolares que puede incidir positivamente en sus hábitos de salud (Gutiérrez y Pino, 2013), se decidió 
adaptar la escala original, orientada a los estudiantes, para conocer la intención de ser físicamente activo en una población adulta en el ámbito laboral de la educación. Para ello ha sido necesario validar la escala tras la adaptación de los ítems 2, 3 y 4 (Tabla1).

Respecto al "Grado de Actividad Física", para medir esta variable se ha empleado el cuestionario PACE (Physicianbased Assessment and Counselling for Exercise) de MartínezGómez et al. (2009). Este cuestionario valora, con dos preguntas, cuántos días en la última semana y en una semana habitual, se realiza al menos, 60 minutos de actividad física. Este instrumento se ha utilizado en otros estudios, en concreto, con adolescentes (López, Valdivielso, Ojeda, Brito, Ruiz y Navarro, 2012) y con adultos (Rodríguez-Larrubia, Aguilar y Pérez, 2011).

Las características sociodemográficas y profesionales de la muestra se recogieron a través de preguntas cerradas.

\section{Análisis estadístico}

Inicialmente, se analizó la validez y fiabilidad de los cuestionarios MIFAM y PACE, seguidamente se calcularon los descriptivos básicos de estos cuestionarios en función de las variables sociodemográficas. Por último, se estudiaron las posibles diferencias en las puntuaciones finales de los cuestionarios en función de las variables sociodemográficas. Dado que no cumplían el supuesto de normalidad se decidió utilizar pruebas no paramétricas para su análisis. Los análisis se realizaron con el software Satistical Package for the Social Sciences versión 21, aplicándose un nivel de significación de 0.05 .

\section{Resultados}

\section{Análisis factorial exploratorio}

Se realizó un análisis factorial exploratorio de componente principales con el objetivo de estudiar la estructura factorial de la escala MIFAM. En primer lugar, se calculó el índice de adecuación muestral de Kaiser-Meyer-Olkin, KMO, que mostró un valor de .851, y también se realizó el test de esfericidad de Bartlett que resultó estadísticamente significativo $\left(\chi^{2}=2177,67 ; p<.001\right)$. Por otro lado, la adecuación a la muestra para cada variable (MSA) indicó que existía fuerza de asociación entre las variables eliminando la influencia de las demás variables, con valores que oscilaron entre $.421 \mathrm{y}$ .874. El valor determinante de la matriz fue .014. La toma en consideración de todos los valores indica que la realización de un análisis factorial es pertinente para estas variables. Tras la realización del análisis factorial se comprobó que los 5 ítems se agrupaban en un único factor: intención de ser físicamente activo. En el análisis factorial resultante, se obtuvo un autovalor de 2,52 y una varianza total explicada de $74.89 \%$ (ver Tabla 2).

Para el análisis factorial confirmatorio se utilizó el método ULS, ya que los datos no cumplieron el supuesto de normalidad multivariante. La bondad de ajuste de los modelos propuestos se evaluó mediante diversos indicadores de ajuste: el índice de bondad de ajuste (Goodness of Fit Index, GFI =.998), el índice de bondad de ajuste corregido (Adjusted Goodness of Fit Index, AGFI = .993), el índice de ajuste normativo (Normed Fit Index, NFI = .996) y el promedio de los residuales (Root Mean Square Residual, RMR = .086). El cuestionario mostró una fiabilidad muy alta con un Alpha de Crombach = .91 (Nunnally, 1978).

El ítem que presentó una mayor puntuación fue el ítem 1, mientras que el que presentó una puntuación menor fue el ítem 3. La puntación media del cuestionario MIFAM fue 3,33 con una desviación típica de 1,35.

Tabla 2. Análisis factorial exploratorio y descriptivos de cada ítem.

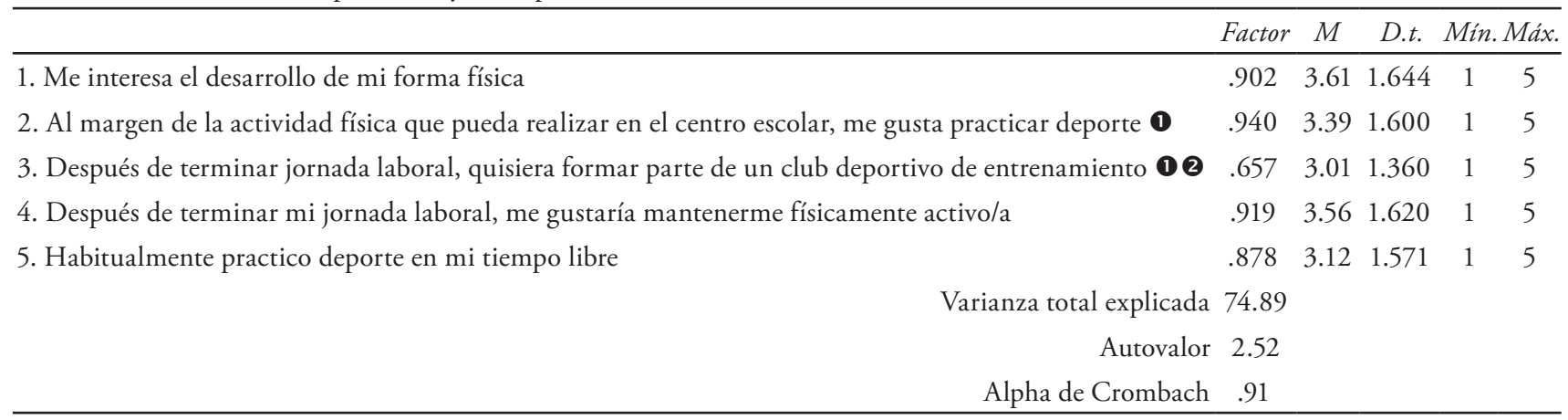


A través del cuestionario PACE (ver tabla 3) se analizó la actividad física semanal de cada maestro/a. Los dos ítems utilizados presentaron una alta fiabilidad, Alpha de Crombach $=.93$ (Nunnally, 1978). Los resultados de la muestra indican que los maestros/as no llegan a tres sesiones semanales $(M=$ $2.66 \pm 1.70)$. Los maestros que más actividad física realizaban tendían a una mayor intención a realizar actividad física en el futuro $\left(r_{\mathrm{o}}=.220, p<.01\right)$.

Tabla 3. Descriptivos del cuestionario PACE.

\begin{tabular}{llll}
\hline & $\mathrm{N}$ & $M$ & D.t. \\
\hline PACE 1. En los últimos 7 días, ¿Cuántos días & 515 & 2.57 & 1.77 \\
hiciste actividad física 60 minutos o más? & & & \\
PACE 2. En una semana normal, ¿Cuántos & 515 & 2.75 & 1.73 \\
días haces actividad física 60 minutos o más? & & & \\
PACE & 515 & 2.66 & 1.70 \\
MIFAM & 515 & 3.33 & 1.35 \\
\hline${ }^{* *} p<.01$ & & &
\end{tabular}

Por otra parte, se analizó la práctica de actividad física semanal en función de las variables sociodemográficas (ver tabla 4) mediante el cuestionario PACE. Los resultados indican que las mujeres realizan menos actividad física que los hombres $(p<.01)$ y que los individuos solteros realizan más actividad física que los que viven en pareja o están casados ( $p<.01)$. En cuanto a la especialidad de los maestros los que ejercen como docentes de educación física presentan mayores valores de práctica física que los maestros de educación primaria e infantil ( $p<.01)$.

Por otro lado, el cuestionario MIFAM (ver tabla 4) presentó diferencias significativas en función de las variables estado civil $(p<.05)$, aunque por los ajustes de la corrección de bonferroni no se pudieron determinar entre qué grupos se encuentran esas diferencias. No obstante, los resultados de la media indican una mayor puntuación en la escala MIFAM en los viudos $(M=4.24 \pm .71)$ y solteros $(M=3.47 \pm 1,41)$, y una menor intención de hacer actividad física en los separados y divorciados $(M=2.97 \pm 1,12)$. Las variables género, edad, tipo de centro, estatus laboral, experiencia y especialidad no presentan diferencias significativas ( $p>.05)$ en las puntuaciones de la escala MIFAM.

Tabla 4. Descriptivos y análisis de diferencias de las puntuaciones finales de los cuestionarios PACE y MIFAM.

\begin{tabular}{|c|c|c|c|c|c|c|c|c|}
\hline & \multicolumn{4}{|c|}{ PACE } & \multicolumn{4}{|c|}{ MIFAM } \\
\hline & $M$ & D.T. & $U$ & $X 2$ & $M$ & D.T. & $U$ & $X 2$ \\
\hline Género & & & $23476.50^{* *}$ & - & & & 28034.00 & - \\
\hline a. Hombre & 3.06 & 1.82 & $a>b$ & & 3.31 & 1.50 & & \\
\hline b. Mujer & 2.47 & 1.61 & & & 3.35 & 1.27 & & \\
\hline Edad & & & - & 1.431 & & & - & 1.737 \\
\hline a. $<25$ años & 2.83 & 1.96 & & & 3.16 & 1.42 & & \\
\hline b. 26 - 40 años & 2.62 & 1.66 & & & 3.38 & 1.37 & & \\
\hline c. 41 - 55 años & 2.74 & 1.69 & & & 3.26 & 1.35 & & \\
\hline d. $<55$ años & 2.40 & 1.73 & & & 3.33 & 1.10 & & \\
\hline Estado Civil & & & - & $14.167^{* *}$ & & & & $9.348^{*}$ \\
\hline a. Soltero & 3.04 & 1.71 & & $a>b$ & 3.47 & 1.41 & & \\
\hline b. Casado/Pareja & 2.47 & 1.67 & & & 3.26 & 1.32 & & \\
\hline c. Divorciado & 2.21 & 1.43 & & & 2.97 & 1.12 & & \\
\hline d. Viudo & 2.00 & 1.87 & & & 4.24 & .71 & & \\
\hline No hijos & & & $26.216^{* *}$ & & & & $28.319^{* *}$ & \\
\hline a. Sin hijos & 2.96 & 1.67 & $a>b$ & & 3.48 & 1.37 & $a>b$ & \\
\hline b. Con hijos & 2.33 & 1.67 & & & 3.18 & 1.31 & & \\
\hline \multicolumn{9}{|l|}{ Tipo de centro } \\
\hline a. Público & 2.67 & 1.64 & 26738.50 & & 3.28 & 1.33 & 29784.00 & \\
\hline b. Concertado & 2.63 & 1.84 & & & 3.48 & 1.39 & & \\
\hline Estatus laboral & & & - & 3.674 & & & & 3.813 \\
\hline a. Funcionario & 2.57 & 1.68 & & & 3.24 & 1.34 & & \\
\hline b. Interino & 2.89 & 1.50 & & & 3.36 & 1.36 & & \\
\hline
\end{tabular}




\begin{tabular}{|c|c|c|c|c|c|c|c|c|}
\hline & \multicolumn{4}{|c|}{ PACE } & \multicolumn{4}{|c|}{ MIFAM } \\
\hline & $M$ & $\overline{D . T .}$ & $U$ & $X 2$ & $M$ & D.T. & $U$ & $X 2$ \\
\hline c. Cooperativista & 3.07 & 1.69 & & & 4.06 & .49 & & \\
\hline d. Contrato indefinido & 2.65 & 1.88 & & & 3.46 & 1.34 & & \\
\hline e. Contrato temporal & 2.56 & 1.78 & & & 3.40 & 1.49 & & \\
\hline Experiencia & & & - & 4.634 & & & & .670 \\
\hline a. $<$ 1año & 2.32 & 1.64 & & & 3.36 & 1.45 & & \\
\hline b. De 1 a 5 años & 2.87 & 1.74 & & & 3.39 & 1.37 & & \\
\hline c. De 5 - 10 años & 2.74 & 1.63 & & & 3.31 & 1.38 & & \\
\hline d. > 10 años & 2.52 & 1.71 & & & 3.31 & 1.33 & & \\
\hline Especialidad & & & & $21.080^{* *}$ & & & & 8.465 \\
\hline a. Ed. Infantil & 2.44 & 1.52 & & $\mathrm{a}<\mathrm{d}^{* *}$ & 3.44 & 1.31 & & \\
\hline b. Ed. Primaria & 2.42 & 1.66 & & $\mathrm{~b}<\mathrm{d}^{* *}$ & 3.24 & 1.35 & & \\
\hline c. Ed. Musical & 2.95 & 1.84 & & & 3.58 & 1.28 & & \\
\hline d. Ed. Física & 3.55 & 1.67 & & & 3.55 & 1.67 & & \\
\hline e. Ed. Especial & 2.83 & 1.56 & & & 3.05 & 1.29 & & \\
\hline f. Lenguas extranjeras & 2.58 & 1.75 & & & 3.49 & 1.23 & & \\
\hline g. Audición y Lenguaje & 2.94 & 1.94 & & & 2.92 & 1.36 & & \\
\hline h. Religión & 2.69 & 1.85 & & & 3.32 & 1.26 & & \\
\hline
\end{tabular}

\section{Discusión}

En este estudio, se planteó como primer objetivo conocer cuál era el grado de práctica físico deportiva de las maestras y maestros en el momento de realizar esta investigación. Nuestros resultados indican que los docentes de educación primaria e infantil realizan menos de tres horas de práctica física semanal, actividad que se encuentra por debajo de las recomendaciones mínimas de organismos internacionales para mantener la salud (Pate, et al., 1995). Son también inferiores a las recomendaciones que los expertos sitúan en torno a los de 30 minutos de actividad física diaria moderada para los adultos al menos 5 días a la semana (Córdoba, et al., 2012), e incluso inferior a los datos que indican que un $57 \%$ de la población española practica deporte tres o más veces por semana (García y Llopis, 2011).

En los resultados se aprecia que esta práctica es mucho menor en las mujeres, observándose además que el estado civil y tener hijos condiciona la posibilidad de realizar práctica física en el tiempo de ocio. Las diferencias de género aquí encontradas coinciden con los resultados obtenidos por García y Llopis (2011) en la encuesta española de hábitos deportivos, donde las mujeres manifiestan un menor interés deportivo, y practican menos deporte que los hombres. En concreto, en dicha encuesta un $31 \%$ de las mujeres manifestaba practicar algún deporte frente al 49\% de los hombres, una diferencia que se ha mantenido estable en las últimas décadas. Este dato arrojado por la encuesta de hábitos deportivos concuerda con lo encontrado en nuestro estudio y puede interpretarse a la luz del tiempo libre del que disponen hombres y mujeres en las primeras etapas de su vida adulta. Las mujeres abandonan con más intensidad la práctica física en esta etapa (cuando acceden al mercado de trabajo y forman una familia) y tienen más de un hora menos de tiempo libre que los hombres (García y Llopis, 2011). Este dato también estaría en línea con los resultados encontrados aquí en relación al estado civil y los hijos. Las maestras y maestros que no tienen hijos realizan una mayor cantidad de práctica física semanal, en concreto, unas tres horas aproximadamente. Además, los solteros son los únicos que llegan a las tres sesiones de práctica física semanal, encontrando diferencias significativas entre estar soltero o vivir en pareja. Por tanto, el tiempo que requiere mantener unos lazos de pareja y un núcleo familiar repercute negativamente en el tiempo dedicado a la actividad física. Y dado que el peso del hogar sigue recayendo en mayor medida en las mujeres no resulta sorprendente encontrar diferencias de género en la práctica física.

En el ámbito laboral se ha podido observar que la única características que parece estar asociada a la práctica de actividad física es la especialidad. En concreto, los maestros de educación física son los que más práctica física realizan en su tiempo de ocio, una tres horas y media, seguidos de los maestros de música con unas tres horas. Los maestros de primaria e infantil son los que menos actividad física realizan, y esta diferencia es significativa en comparación con los maestros de la especialidad de educación física. 
La intencionalidad de ser físicamente activo de los maestros fue media $(M=3.33 \pm 1.35)$, en una escala de cinco pasos, y esta se sitúa por debajo de la intención declarada por los escolares de educación primaria con una media de 3.94 \pm 1.13 (Arias et al., 2013), de alumnos de educación secundaria, $M=3.96 \pm 1.26$ (Moreno et al., 2007), de jugadores de baloncesto en edad escolar, $\mathrm{M}=4.19 \pm .69$ (Franco, et al., 2012) y de los universitarios, $M=4.58 \pm .47$ (Expósito, et al, 2012). Las características sociodemográficas personales, como el estado civil y el número de hijos influyen en la intención de realizar práctica física en el futuro. Los docentes solteros/as y sin hijos puntúan más alto en la intención de hacer actividad física.

Los maestros y maestras que más actividad física realizan presentan una tendencia a una mayor intención a realizar actividad física en el futuro. Con lo cual la intención es un buen predictor del comportamiento, tal y como señalaba Hein, et al. (2004). Esto significa que trabajar en el binomio motivación/intención repercute positivamente en la práctica real que se acaba llevando a la práctica y que, por tanto, realizar campañas de motivación entre los maestros que presentan bajos niveles de actividad física puede ser una buena herramienta para cambiar sus hábitos físicos.

El estudio presentado presenta algunas limitaciones que deberían ser consideradas en estudios posteriores. En primer lugar, la medida de práctica física debería ser recogida con un cuestionario más profundo que permita equiparar los resultados con otras poblaciones. Además, se podrían tomar algunas medidas directas e indirectas de la condición de salud.

\section{Aplicaciones prácticas}

Existen evidencias científicas entre salud y práctica de ejercicio físico, siendo el sedentarismo un agente altamente nocivo.

Este estudio muestra como en el colectivo profesional de los maestros en España la práctica de ejercicio físico semanal se encuentra por debajo de las recomendaciones mínimas de organismos internacionales, que dicha incidencia es mayor en mujeres; y donde el estado civil así como el hecho de tener hijos, se constituyen en variables fundamentales de influencia en la práctica motriz. De igual modo, se muestra que la intención de práctica de ejercicio físico es un buen predictor y que los maestros que más práctica de ejercicio físico realizan son aquellos de la especialidad de Educación Física. En aras a mejorar esta situación, el binomio motivación/intención; puede ser una clave fundamental. Esto llevaría a aplicar una de las teorías más utilizadas en la promoción de la salud y con gran influencia del componente cognitivo/perceptivo: el Modelo de Creencias de Salud (Becker, 1974). Dicho planteamiento descansa sobre la hipótesis de que la consecución de una meta depende del valor que el sujeto atribuye a la misma y la probabilidad de que las acciones realizadas sean eficientes para su consecución.

Desde este enfoque, se haría muy necesario en el colectivo de maestros la adopción de acciones más efectivas de concienciación sobre el riesgo que supone la inactividad física (concienciar del valor), así como la necesidad de organización en su vida doméstica para conquistar tiempo y destinarlo a su realización (probabilidad de logro); ya que el impacto del cuidado de hijos y gestión doméstica parece tener una gran influencia en el descenso de práctica.

\section{Referencias}

1. Arias, J.L., Castejón, F.J. y Yuste, L. (2013). Propiedades psicométricas de la escala de intencionalidad de ser físicamente activo en Educación Primaria. Revista de Educación, 362, 485-505.

2. Ato, M., López, J.J. y Benavente, A. (2013). Un sistema de clasificación de los diseños de investigación en psicología. Anales de psicología, 29(3), 1038-1059.

3. Becker, M.H. (1974). The health belief model and personal health behavior. Health Educ Monogr, 2(4), 324-508.

4. Caracuel, J.C. y Arbinaga, F. (2010). Repercusión del ejercicio físico sobre la salud. Apuntes de psicología, 28(2), 315-328.

5. Córdoba, R., Cabezas, C., Camarelles, F., Gómez, J., Díaz, D., López, A., Marqués, F. Muñoz, E., Navarro, B., Ramírez, J.I. (2012). Recomendaciones sobre el estilo de vida. Atención Primaria, 44(1), 16-22.

6. Expósito, C., Fernández E.J., Almagro B.J. y Sáenz-López, P. (2012). Validación de la escala medida de la intencionalidad para ser físicamente activo adaptada al contexto universitario. Cuadernos de Psicología del Deporte, 12 (2), 49-56.

7. Fox, K.R. (1999). The influence of physical activity on mental wellbeing, Public Health Nutrition, 2(3), 411-418

8. Franco, E., Pérez-Tejero, J. y Arrizabalaga, A. (2012). Motivación e intención de ser físicamente activo en jugadores de baloncesto en forma- ción. Diferencias en función de la competición. Cuadernos de Psicología del Deporte, 12(1), 23-26.

9. García, M. y Llopis, R. (2011). Encuesta sobre los hábitos deportivos en España 2010. Madrid: Consejo Superior de Deportes y Centro de Investigaciones Sociológicas. Recuperada de: http://www.csd.gob.es/csd/ estaticos/dep-soc/encuesta-habitos-deportivos2010.pdf.

10. Gutiérrez, A. y Pino, M. (2013). Actitudes hacia la práctica de actividad física saludable en futuros docentes. Cuadernos de Psicología del Deporte, 13(2), 73-82

11. Hein, V., Müür, M., y Koka, A. (2004). Intention to be physically active after school graduation and its relationship to three types of intrinsic motivation. European Physical Education Review, 10(1), 5-19.

12. INE (2013). Presentación de la encuesta nacional de salud: ENSE 2011-2012. Recuperado de: https://www.msssi.gob.es/estadEstudios/ estadisticas/encuestaNacional/encuesta2011.htm.

13. Martínez-Gómez, D., Martínez-De-Haro, V., Del-Campo, J., Zapatera, B., Welk, G., Villagra, A., et al. (2009). Validez de cuatro cuestionarios para valorar la actividad física en adolescentes españoles. Gaceta Sanitaria, 23(6), 512-517.

14. Martínez, S., Rodríguez, J., y De Abajo, S. (2008). Sedentarismo y salud: efectos beneficiosos de la actividad física. Apunts: Educación física y Deportes, 83, 12-24. 
15. Moreno, J.A., Moreno, R. y Cervelló, E. (2007). El autoconcepto físico como predictor de la intención de ser físicamente activo. Psicología y Salud, 17(2) 261-267.

16. Moscoso, D.; Serrano, R.; Biedma, L. y Martín, M. (2013). Ciudadanía activa versus ciudadanía sedentaria. Un nuevo canon social en el acceso a la salud y el bienestar. Methaodos. Revista de Ciencias Sociales, 1(1), 123-140.

17. Nocon, M., Hiemann, T., Müller-Riemenschneider, F., Thalau, F., Roll, S. y Willich, S.N. (2008). Association of physical activity with all-cause and cardiovascular mortality: a systematic review and metaanalysis. The European Journal of Cardiovascular Prevention and Rehabilitation, 15(3), 239-46.

18. OMS (2009). 2008-2013 Action Plan for the Global Strategy for the Prevention and Control of Noncommunicable Diseases. Recuperado de: http://www.who.int/nmh/publications/9789241597418/en/

19. OMS (2010). Global status report on noncommunicable diseases, 2010. Recuperado de: http://www.who.int/nmh/publications/ncd_report_full_en.pdf

20. Pate, R.R., Pratt, M., Blair, S.N., Haskell, W.L., Macera, C.A., Bou- chard, C. et al (1995). Physical activity and public health. A recommendation from the Centers for Disease Control and Prevention and the American College of Sports Medicine. JAMA, 273, 402-407.

21. Ramírez, A., Timón, R., Olcina, G., Muñoz, D., Robles, M.C., Maynar, M. (2012). Los efectos de un programa de actividad física aeróbica sobre el estado de ánimo en mujeres premenopáusicas. E-balonmano. com: Revista de Ciencias del Deporte, 8(2), 121-130.

22. Rodríguez-Larrubia, P., Aguilar, J.M. y Pérez, E. (2011). Influencia de la actividad física sobre los niveles de depresión. International Journal of Developmental and Educational Psychology, 5(1), 203-208.

23. Sofi, F., Capalbo, A., Cesari, F., Abbate, R. y Gensini, G.F. (2008). Physical activity during leisure time and primary prevention of coronary heart disease: an updated meta-analysis of cohort studies. The European Journal of Cardiovascular Prevention and Rehabilitation, 15(3), 247-57. doi: 10.1097/HJR.0b013e3282f232ac.

24. Trost, S.G., Pate, R., Freedson, P.S., Sallis, J.F. y Taylor, W.C. (2000). Using objective physical activity measures with youth: How many days or monitoring are needed? Medicine and Science in Sports and Exercise, 32, 426-431. 